\title{
BMJ Open Which features of primary care affect unscheduled secondary care use? A systematic review
}

\author{
Alyson Huntley, ${ }^{1}$ Daniel Lasserson, ${ }^{2}$ Lesley Wye, ${ }^{1}$ Richard Morris, ${ }^{3}$ \\ Kath Checkland, ${ }^{4}$ Helen England, ${ }^{1}$ Chris Salisbury, ${ }^{1}$ Sarah Purdy ${ }^{1}$
}

To cite: Huntley A, Lasserson D, Wye L, et al. Which features of primary care affect unscheduled secondary care use? A systematic review. BMJ Open 2014;4:e004746. doi:10.1136/bmjopen-2013004746

- Prepublication history and additional material is available. To view please visit the journal (http://dx.doi.org/ 10.1136/bmjopen-2013004746).

Received 30 December 2013 Revised 16 April 2014 Accepted 23 April 2014

\section{CrossMark}

${ }^{1}$ School of Community \& Social Medicine, Centre of Academic Primary Care, University of Bristol, Bristol, UK

${ }^{2}$ Department of Primary Care Health Sciences, University of Oxford, Oxford, UK

${ }^{3}$ Primary Care \& Population Health, Royal Free Campus,

London, UK

${ }^{4}$ Institute of Population Health, Centre for Primary

Care, University of

Manchester, Manchester, UK

Correspondence to

Sarah Purdy;

sarah.purdy@bristol.ac.uk

\begin{abstract}
Objectives: To conduct a systematic review to identify studies that describe factors and interventions at primary care practice level that impact on levels of utilisation of unscheduled secondary care.

Setting: Observational studies at primary care practice level.

Participants: Studies included people of any age of either sex living in Organisation for Economic Cooperation and Development $(\mathrm{OECD})$ countries with any health condition.
\end{abstract}

Primary and secondary outcome measures: The primary outcome measure was unscheduled secondary care as measured by emergency department attendance and emergency hospital admissions.

Results: 48 papers were identified describing potential influencing features on emergency department visits ( $n=24$ studies) and emergency admissions $(n=22$ studies). Patient factors associated with both outcomes were increased age, reduced socioeconomic status, lower educational attainment, chronic disease and multimorbidity. Features of primary care affecting unscheduled secondary care were more complex. Being able to see the same healthcare professional reduced unscheduled secondary care. Generally, better access was associated with reduced unscheduled care in the USA. Proximity to healthcare provision influenced patterns of use. Evidence relating to quality of care was limited and mixed.

Conclusions: The majority of research was from different healthcare systems and limited in the extent to which it can inform policy. However, there is evidence that continuity of care is associated with reduced emergency department attendance and emergency hospital admissions.

\section{INTRODUCTION}

Unscheduled care is defined as any unplanned contact with the health service by a person requiring or seeking help, care or advice. ${ }^{1}$ It includes urgent care which comprises conditions that require assessment and planned intervention within 7 days, or which is likely to lead to an emergency within 4 weeks and emergency care which is not

\section{Strengths and limitations of this study}

- This review was conducted following rigorous Cochrane methodology.

- We included studies published after 2000 and conducted in Organisation for Economic Co-operation and Development countries to ensure that the results are as relevant as possible to primary healthcare provision in developed countries.

- Seven of the 44 studies had univariable analysis, which limits the interpretation of results.

- Although some studies were countrywide, others were concerned with more discrete populations, making generalisation more difficult.

- Research was carried out in different healthcare systems; findings from one setting may not be generalisable to other settings.

always life-threatening but needs prompt assessment and a planned intervention within 24 hours. $^{2}$ There are five levels of unscheduled healthcare from self-care (level 1), primary care, minor injury unit, etc (level 2) through to level 3 (emergency department (ED)) and hospital admission (level 4) and specialised hospital support (level 5). ${ }^{3}$

Reducing unscheduled care use in the secondary care sector (ie, ED attendance and emergency hospital admission (EHA)) is a priority for many healthcare systems. For example, in a recent King's Fund report, it was suggested that emergency admissions among people with long-term conditions that could have been managed in primary care cost the National Health Service (NHS) $£ 1.42$ billion annually and that this could be reduced by $8-18 \%$ through investment in primary and community-based services. ${ }^{4}$

Patterns of attendance at ED vary according to the local healthcare system and population but overall attendances at ED are rising. ${ }^{5}$ In the UK, despite the universal provision of primary care for which there is no charge at the point of access, there were 
17.6 million ED attendances recorded at major ED departments, single specialty A\&E departments, walk-in centres and minor injury units in England in 2011-2012: an increase of $8.5 \%$ from 2010 to $2011 .{ }^{6}$ In the UK, many patients use ED even when primary care offices or practices are open, with weekday attendances peaking in the mid-morning.

There is considerable variation in emergency admission and ED attendance rates across general practices. ${ }^{78}$ The reason for this variation in rates across practice is poorly understood. Previous systematic reviews have been limited to looking at access and continuity of care. ${ }^{9-13}$ The reviews of primary care access described the effect on ED use and not EHA, and included data in the continuity of care reviews were over 5 years old. This is the first review, to the best of our knowledge, that synthesises the effect of patient features, primary care access, features of primary care practice, continuity of care and quality markers on both ED use and EHA.

Our objective therefore was to conduct a systematic review to identify studies that describe features of primary care services that impact on levels of utilisation of unscheduled secondary healthcare (USC) (see online supplementary data-protocol).

\section{METHODS}

A systematic review was conducted to identify studies that describe factors at primary care practice level that impact on levels of utilisation of USC.

\section{Inclusion criteria}

\section{Study population}

Studies that included people of any age of either sex living in Organisation for Economic Co-operation and Development (OECD) countries. ${ }^{14}$

\section{Types of features of primary healthcare}

For both in hours and out of hours services, we selected studies examining the impact of patient features, access to primary healthcare, features of the practice, continuity of care and quality markers.

\section{Types of studies}

Observational studies about features of primary care services associated with unscheduled secondary care use. We included studies written in any language.

\section{Outcomes}

Any studies concerning any health condition as long as the outcome of interest was utilisation of USC, that is, attendance at an ED or an EHA.

\section{Exclusion criteria}

Studies that only reported admission for elective or planned healthcare including planned diagnostic services, admission to a community or non-acute hospital as an outcome and clinical trials primarily about the management of conditions. We excluded pre-2000 studies as primary healthcare provision has changed significantly over time and older studies were less relevant.

\section{Searches and reference management}

A search strategy was developed in Medline for the electronic databases according to their specific subject headings or searching structure to search for papers describing both primary studies and systematic reviews (see online supplementary data-search strategy). Other databases searched were EMBASE, CINAHL, PsycINFO and the Cochrane Library. All databases were searched from inception until October 2012. This review was part of a wider group of reviews that also included interventional and qualitative studies; thus, the search strategy and the PRISMA flow chart reflect this (see online supplementary data—search strategy; figure 1).

The search strategy was modified to search internet sites such as the Agency for Healthcare Research and Quality and the King's Fund. Reference lists of included papers were checked for further potentially eligible studies. These references underwent a two-stage process of screening using the inclusion and exclusion criteria by two independent reviewers. The first screen was of titles and abstracts and the second of the full papers. Where there was continued disagreement between reviewers about including or excluding a paper, a third reviewer made the final decision.

\section{Data collection, analysis and reporting}

Standardised data extraction forms were developed and then data were abstracted by one reviewer and a second reviewer checked data abstraction against the original paper. During this process, we divided the data into patient features, access to primary healthcare, features of the practice, continuity of care and quality markers. Quality of studies was assessed by two reviewers using a modified CASP appraisal tool for cohort and case control studies as appropriate for all the included studies. ${ }^{15}$

\section{RESULTS}

\section{General}

We identified 48 papers relevant to the aim of our study; 24 studies of features influencing ED visits and 22 studies of features influencing $\mathrm{EHA}^{16-59}$ (figure 1). This included one paper describing one study with both outcomes, ${ }^{16}$ two sister papers describing ED visits and EHA, respectively, within the same population ${ }^{7} 17$ and two papers describing the same study on EHA. ${ }^{18} 19$ Thus, the review contained 44 individual studies of which the majority of studies were cross-sectional in design $(n=38)$. The remaining studies were a mixture of designs: longitudinal $(n=2)$, before and after $(n=1)$ and case control $(n=2)$.Generally, the papers described data across two or more features of primary healthcare. 
Figure 1 PRISMA flow diagram.

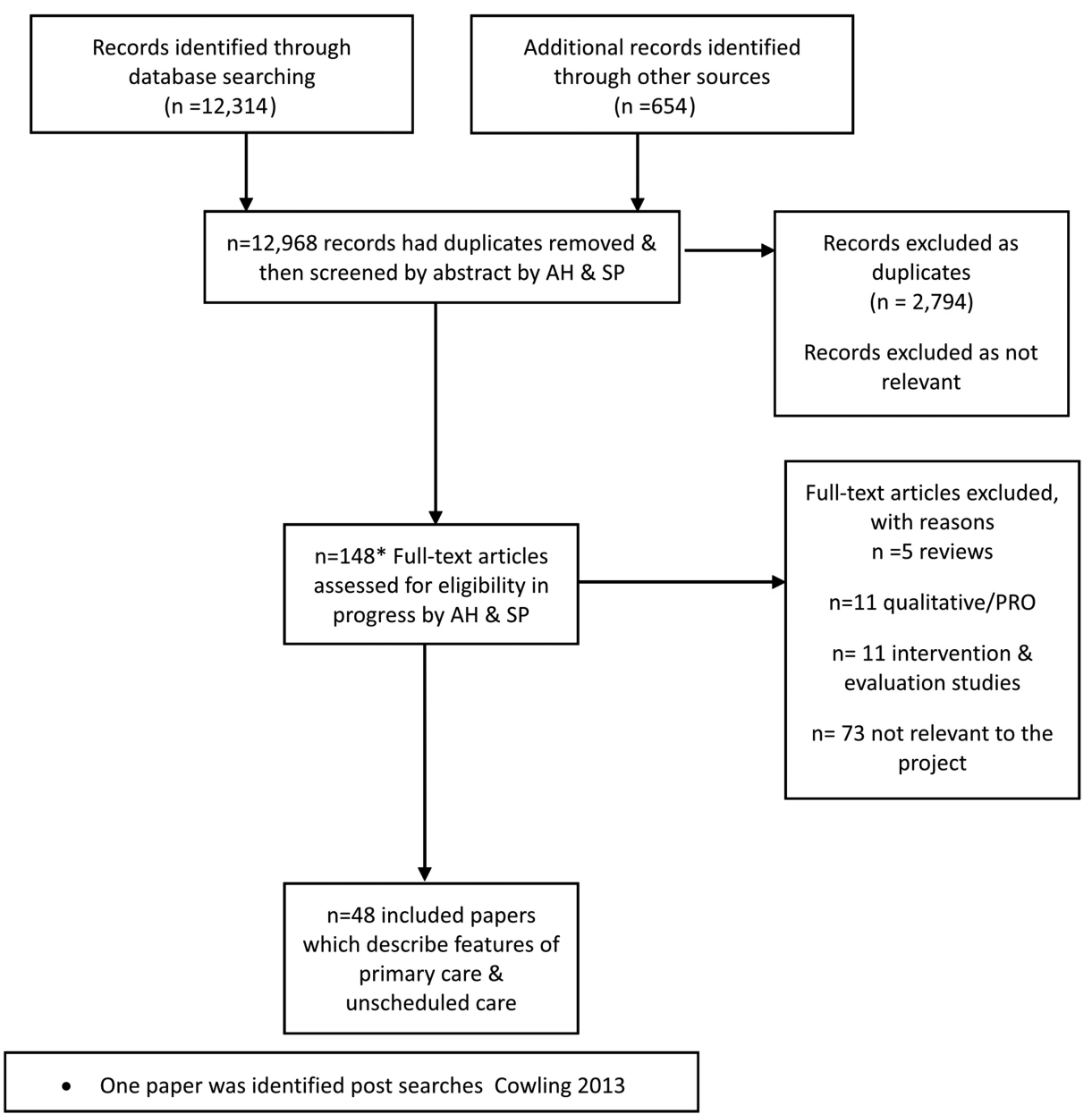

\section{Quality appraisal}

Several issues came out of the CASP quality appraisal of the studies (see online supplementary data-CASP). One was the generalisability of the studies; while some studies were country or state-wide for the whole population, ${ }^{20-22}$ others analysed much smaller populations, for example, inner city boroughs. ${ }^{23}{ }^{24}$ Equally, some studies involved all patients on GP lists ${ }^{7}$ while others dealt with specific groups such as patients with diabetes or paediatric patients. $^{26-28}$

The majority of the studies described problems with response rates, bias and confounding factors. For some studies, this imposed serious caveats about the findings from the study, for example, poor response rates ${ }^{27}$ or only univariable analysis was performed (seven studies, see online supplementary data-CASP). ${ }^{7} \begin{array}{lllll}16 & 25 & 27 & 29-31\end{array}$ The majority of studies performed multivariable analysis and so attempted to adjust for confounding factors. Only four studies described cost data, while the majority of the studies merely stated that the results had cost implications. $^{20} 32-34$

\section{Impact of patient characteristics on unscheduled care ( $n=21$ studies)}

The effect of patient characteristics on unscheduled care was described in 22 papers (21 studies), and this was usually in combination with investigation of nonpatient factors (see online supplementary data-tables $1 \mathrm{ab}$ and $2 \mathrm{ab})$. There were $\mathrm{n}=8$ investigating ED use and $\mathrm{n}=14$ investigating EHA and the 21 studies were spread across the UK $(n=11)$, the USA $(n=5)$, South America $(n=1)$, Canada $(n=1)$, Norway $(n=1)$, Italy $(n=1)$ and Spain $(n=1)$.

Age, gender and ethnicity

All studies show that increased age is associated with increased ED attendance ${ }^{28} \quad 35$ and increased EHA. $^{17} 34$ 36-38 The only exception was Cowling 2013, a study covering $95 \%$ of GP practices in England, which showed that an increase in the percentage of patients aged 65 years or older was associated with a small reduction in patients who self-referred to, and were then subsequently discharged from ED (relative rate $0.989(95 \%$ CI 0.984 to 0.994$), \mathrm{p}<0.001)$, suggesting that older patients may be less likely to attend with minor illness. ${ }^{39}$ However, gender appears to be less important in ED attendance with four studies showing no effect with gender.7 283940

The evidence about the impact of gender on EHA is mixed with two studies from the UK and Norway showing that women are more likely to experience $\mathrm{EHA}^{17} 34$ and three studies from Italy, Spain and the 
USA showing that men are more likely to undergo EHA. $^{37} 38$ It is therefore possible that these effects are country/or culture specific.

The evidence for the effect of ethnicity is also mixed for $\mathrm{ED}$ attendance ${ }^{7} \quad 28 \quad 30 \quad 40$ and EHA. ${ }^{35} \quad 37 \quad 38 \quad 42 \quad 45$ However, this may be due to the lack of data on ethnicity admissions and dependency on location and ethnic mix of population.

\section{Socioeconomic status}

Decreased socioeconomic status is consistently associated

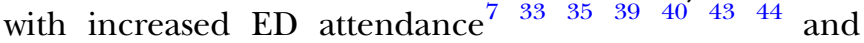
increased EHA. 816172122253645 A similar effect is seen with social isolation and lack of social support for ED attendance $^{3540}$ and EHA. ${ }^{83541}$

One study associated increased education with reduced ED attendance. ${ }^{26}$ Increased education is consistently associated with reduced EHA. ${ }^{34} 4145$

In the USA, insurance status was associated with unscheduled care use. Two studies showed that adult Medicaid patients use the ED more and have more EHA than private insurance patients. ${ }^{18} 38$ Another US study showed that parents of children with public health insurance who perceive good family centredness in their primary healthcare provision had reduced ED attendance. ${ }^{28}$ In the UK, a study by Harris with multivariable analysis of data from 68 practices from Brent (North London) primary care trust (PCT) suggests that for a population that is older, male, white and living alone, being on a GP register as opposed to having no GP has no effect on ED use. ${ }^{40}$ Brent PCT is an inner-London borough characterised by its ethnic diversity and high levels of deprivation.

\section{Health state}

Having a chronic disease or multimorbidity is associated with ED attendance and EHA. One study showed that underlying morbidity in the presence of cardiovascular disease, or digestive disease, is associated with increased ED attendance, as are terminal illness and overall comorbidity. ${ }^{35}$ This study also showed that an increased number of days in hospitals is associated with subsequent increased ED attendance. ${ }^{35}$

The presence of chronic diseases coronary artery disease (CHD), angina, asthma and chronic obstructive pulmonary disease (COPD) has been associated with $\mathrm{EHA}^{22}{ }^{22} 46$ as is the combination of smoking with CHD, asthma or COPD. ${ }^{21}{ }^{22}$ However, Cowling 2013 found no effect of the prevalence of asthma, obesity and hypertension in English practice on ED visits. ${ }^{39}$ Age-standardised patient increased mortality rates are also associated with increased EHA. ${ }^{8} 35$

There is one study associating worse self-reported health and increased EHA. ${ }^{37}$ This same study found a greater number of hospital admissions following lower primary healthcare use in a period of 1 year and that shorter previous hospital stays resulted in increased subsequent EHA. ${ }^{37}$
ED attendance studies and features of primary healthcare provision $(\mathrm{n}=24)$

More than half of these studies $(n=16)$ were conducted in the US A and Canada. The majority of the studies are cross-sectional in design $(n=19)$. These studies are described in detail in online supplementary data-table $1 \mathrm{a}$ and the main results are summarised in online supplementary data-table $1 b$.

\section{Access}

Four US studies and one UK study indicate that increased access to primary care in terms of longer opening hours, more appointment slots available and increased nurse triage reduce ED attendance. 272839434447 The UK study is based on 7885 primary care practices and suggests that general practices providing more timely access to primary care had fewer self-referred discharged ED visits per registered patient. ${ }^{39}$ One of the US studies suggests that this is true for both public and private insurance patients. $^{28}$

One study in the Netherlands showed that positioning GP out of hours clinics near EDs reduced ED attendance. ${ }^{48}$ However, changes to the delivery of out of hours primary healthcare in the UK since 2004 have transferred responsibility for out of hours care from practices to the local PCT which provides care across a local geographical area. One longitudinal study has shown that this change has increased ED use at a UK district general hospital. ${ }^{29}$ Similarly in Denmark, when out of hours care by local GPs was replaced by telephone triage by GPs in a central regional triage centre and geographically larger rota systems, ED visits increased. ${ }^{49}$ However, one study conducted in Spain reported that greater access to the primary care continuing care points (out of hours) did not have any effects on ED attendance. ${ }^{50}$

$\mathrm{ED}$ attendance is also increased if patients do not have a regular GP 355152 or a specialist practitioner, ${ }^{52}$ although there is a UK study based on data from one PCT in an ethnically diverse and deprived area which suggests that being registered with a GP for patients greater than 65 years did not influence ED use. ${ }^{40}$ The picture is mixed in terms of higher physician to patient ratio influencing ED attendance as one study ${ }^{35}$ showed that high family physician availability was associated with greater ED use, although this study included areas with low specialist availability, which could limit access to more intensive management of ambulatory care sensitive conditions. In two studies, a higher ratio of GPs to registered patients had no effect on certain types of ED usage. ${ }^{35} 39$

Patients' perception of poor primary healthcare access in terms of telephone access, shorter opening hours, no alternative place to seek advice, inability to get appointments and unmet needs was associated with increased ED attendance. 71952

\section{Practice features}

Practice features have an inconsistent association with ED attendance. One UK county-wide study suggests that 
a smaller practice size is associated with increased ED attendance. ${ }^{7}$ This same study also showed that patient proximity to a primary healthcare practice reduced ED attendance. ${ }^{7}$ Another smaller UK study based in a north London district showed that close proximity to primary care practice had no effect on ED attendance. ${ }^{40}$ However, a more recent and larger England-wide study by Cowling suggested that the shorter distance to GP practice compared to distance to hospital by foot or public transport reduced ED attendance. ${ }^{39}$ A US study focused on the paediatric population showed that proximity to a primary healthcare practice reduced ED attendance, but that proximity to ED increased use. ${ }^{53} \mathrm{~A}$ further US study reported that the shorter time to drive to hospital from home was associated with increased ED use. $^{47}$

While practices lacking nebulisers for children and peak flow meters for adults showed increased ED attendance in one US study, this study also found that practices lacking inhalers reduced ED attendance. ${ }^{43}$ The authors suggest that patient behaviour may be affected differently by these devices but could offer no real explanation for these conflicting data. Practices having the same day turnaround of laboratory tests were associated with a reduction in ED attendance. ${ }^{44}$

Practices in the USA with nurse practitioners or physician assistants were associated with increased ED attendance ${ }^{43}$ but a UK study found that if care was provided by either a nurse or a doctor, there was no effect on ED use. ${ }^{40}$ Practices in North America in which at least one clinician made hospital rounds, or which had a specialist physician as opposed to a family physician (for older people) were associated with increased ED attendance. ${ }^{43}{ }^{5}$

\section{Continuity of care}

Five studies, three from the USA and two from Canada, consistently showed that continuity of care as measured by seeing the same family or specialist physician reduced ED attendance. ${ }^{1629355254}$

\section{Quality of care measures}

Overall, there is a paucity of evidence for the relationship between the quality of general practice care and ED attendance; however, one study showed that better quality of care (as measured by use of cholesterol tests, glycated haemoglobin tests, referrals to ophthalmologists and recommendation to stop smoking) for patients with diabetes reduced ED attendance of these patients. ${ }^{26}$

\section{EHA studies and features of primary healthcare provision $(\mathrm{n}=22)$}

The majority of these studies $(n=12)$ were conducted in the UK and cross-sectional in design $(n=21)$. These studies are described in detail in online supplementary data-table $2 \mathrm{a}$ and the main results are summarised in online supplementary data-table $2 \mathrm{~b}$.

\section{Access}

One US study showed that poorer access to primary care services increased EHA, but a study in Ireland showed that increasing free primary care to those patients over 70 years of age had no effect on EHA. ${ }^{55} 56$

While there are five studies from different countries, which suggests that an increase in GP supply (availability of GPs in an area) and a higher ratio of practitioners to patients are associated with reduced admissions, ${ }^{22} 37384557$ there are also five studies that looked at similar measures: physician density, GP per 10000 population, average list per partner, physician supply and percentage of GPs with $>2500$ patients, which showed no effect on admissions. 821343645

\section{Practice features}

The impact of overall size of a GP practice on EHA is conflicting. Evidence from three studies showed training and course provision within GP practices decreased EHA of patients from those practices. ${ }^{21} 2241$

Two studies show that an increased distance of primary care practice from the hospital reduces EHA. ${ }^{17}$ Equally, patient data show that urban dwelling and proximity to hospital increase admissions. ${ }^{21} 2238$ There is evidence that training ( $\mathrm{n}=3$ studies) and course provision ( $\mathrm{n}=1$ studies) within GP practices decrease EHA of patients from those practices. Features that do not appear to reduce EHA are the numbers of GP partners, the number of partners with formal postgraduate qualifications in general practice or the proportion of salaried GPs. ${ }^{34}{ }^{36}$ However, there is one US study which shows that an increase in specialists in primary care is associated with increased EHA. ${ }^{38}$ There is one study that shows that having female GPs in a practice is associated with reduced EHA. ${ }^{41}$

The evidence for practices providing specific services is mixed. One study showed that cervical screening, child health surveillance, emergency contraception and maternity services were associated with increased EHA. ${ }^{42}$ One UK study showed that providing prescription services for asthma, diabetes, heart failure, hypertension and COPD, as well as diabetes and asthma specialist services, has no effect on admissions. ${ }^{8}$

However, the amount of certain services does seem to impact on reducing EHA with both health visitor hours per 1000 children under the age of 5 , and the number of primary care visits in the last months of life in congestive heart failure and patients with COPD being associated with fewer EHA. ${ }^{58} 59$

\section{Continuity of care}

As with ED visits, there is evidence on continuity of care (seeing the same health professional) and EHA. However, the data suggest that the effect may be context and condition-specific. One UK study shows that the easier it is to get an appointment with your own GP, the lower the EHA. ${ }^{17}$ A US study shows that reduced continuity of care with paediatric patients on Medicaid or 
with asthma was associated with increased EHA, and one study carried out in Manitoba, Canada showed that high continuity of care was associated with a reduction in EHA. ${ }^{16}{ }^{23}$ However, one further US study of diabetes, CHD and depression patients suggests that improved continuity of care with the same physician had no effect on EHA. ${ }^{32}$

\section{Quality of care measures}

While two UK studies showed that general performance indicators for primary care practice had no effect on $\mathrm{EHA},{ }^{17} 42$ the evidence for quality of care measures for specific conditions is mixed. For patients with diabetes, two studies show that improved quality indicators reduce $\mathrm{EHA},{ }^{20}{ }^{25}$ but one of these studies suggests that this association is only valid when comparing moderate to poor Quality and Outcomes Framework (QoF) indicators, and that when moderate is compared with high indicators there is no effect. ${ }^{20}$

High quality scores for angina were associated with reduced EHA, but condition-specific quality markers for myocardial infarction (MI) had no effect on EHA. ${ }^{22}$

Diagnosis of asthma by spirometry was shown to be associated with reduced EHA, but there was no effect on EHA for asthmatics who received a review. ${ }^{21}$ There was also no effect on EHA with increased clinical QoF scores for patients with COPD. ${ }^{21}$ Patient satisfaction with primary healthcare services is associated with reduction of EHA. ${ }^{34} 37$

\section{DISCUSSION}

\section{Statement of principal findings}

This review identified 48 papers which described 24 studies of features influencing ED visits, and 22 studies of features influencing EHA. The patient factors influencing unscheduled care were similar for ED use and EHA and were consistent across countries. The most important of these were increased age, reduced socioeconomic status, lower educational achievement and the presence of chronic disease and multimorbidity, which were all associated with increased unscheduled secondary care. In addition, proximity of patients to healthcare provision strongly influences their use despite the country of residence, that is, if they live near an $\mathrm{ED} /$ hospital, they are more likely to use these services than if they live more remotely. Equally, if patients live near a primary care facility, then unscheduled secondary care may be reduced.

The main feature of primary care that is consistently associated with reduced unscheduled care use is continuity of care. Studies from the USA, Canada and the UK suggest that being able to see the same family or specialist practitioner reduces both ED use and EHA. However, the evidence of effect on unscheduled secondary care of increased access to primary healthcare was mixed. In general, better access to primary care was associated with reduced use of ED and EHA in the USA and
Canada. However, the relationship in European health systems is less clear with no clear overall patterns emerging from the identified studies.

Organisational features of primary healthcare affecting ED attendance and EHA are more complex to describe with heterogeneity of findings across healthcare systems and within systems. The evidence for quality of care markers is inconclusive.

\section{Strengths and weaknesses of the study}

This review was conducted following rigorous Cochrane methodology with a focused search conducted in all the major databases. There was no language restriction on the studies retrieved, but the studies were restricted to OECD countries and to those published from 2000 onwards to ensure that the studies were as relevant as possible to the current primary health provision in developed countries.

The initial search only included studies published up to October 2012. An updated limited search before the analysis of included studies identified one paper which was included in the main results section ${ }^{39} \mathrm{~A}$ final updated search identified a further four relevant papers published up to December 2013. Two studies were found from the USA. One cross-sectional study included further evidence that fewer primary care physicians per capita are associated with higher ED attendance rates. ${ }^{60}$ A second paper was a before and after study of the introduction of a patient-centred medical home model across a health system. ${ }^{61}$ This study found a reduction in emergency department visits but not emergency admissions in patients using the new model of care. A further crosssectional study from England suggested that being able to book an appointment with a preferred primary care doctor is associated with fewer admissions. ${ }^{62}$ A second English study found that nationally falling rates of admission for heart failure are not associated with characteristics of primary care, including quality of care.$^{63}$ None of these studies contradict the findings of the initial review.

Seven of the originally included cross-sectional studies only reported univariable analysis, which limits the identification of factors that significantly influence the measures of unscheduled secondary care, as potential confounding factors will not be incorporated in modelling. Definitions of unscheduled secondary care also differed between studies limiting comparisons and synthesis. While some studies were countrywide, many studies were on relatively discrete populations which have may not be generalisable to all the patient groups within a healthcare system. For example, Cowling $e t a l^{39}$ found that having a greater proportion of patients older than 65 years in a practice population was associated with reduced ED attendance. However, the outcome was based on patients who had self-referred and then had been discharged and were therefore likely to be a cohort of patients with minor illness, rather than the 
total cohort presenting to ED which would include those with more significant pathology.

Furthermore, as research was carried out in different healthcare systems, findings from one setting may not be generalisable to other settings. The ED attendance studies were predominantly from the USA and Canada while the majority of the EHA studies were UK-based, and therefore this limits the generalisable conclusions. There were very little cost data or analyses, and so the financial case for implementing services cannot be made from the identified studies.

\section{Strengths and weaknesses in relation to other studies/ important differences in the results}

There are five systematic reviews that are relevant to this current review. ${ }^{9-13}$ Two of the reviews focus on access to primary healthcare and ED use, but there were no data on EHA in either of these reviews. ${ }^{11}{ }^{12}$ Both reviews include worldwide studies and suggest that improved patient access to primary care reduces ED use, but neither review explicitly addressed country-specific health systems and their differing issues.

Three of the reviews focus on continuity of care and unscheduled secondary care. ${ }^{9} 10 \quad 13$ The reviews by Cabana and Hsiao looked at continuity of primary healthcare and unscheduled secondary care, but the data are over 5 years old. However, both these reviews reflect the updated findings of our review, namely that continuity of care reduces unscheduled secondary care. The review by Aubin $e t a l^{9}$ only focuses on patients with cancer and considered studies across primary and secondary care.

\section{Meaning of the study-possible explanations and implications for clinicians and policymakers}

While the expected associations with increased ED use were seen for patient level factors that are associated with greater prevalence of acute illness (increasing age, indices of low socioeconomic status, chronic disease), there were few clear overall associations across the published evidence for primary care practice or healthcare system factors. This is likely to be due to the importance of the background healthcare context such as insurance based systems without universal health coverage or healthcare with free access at the point of use. Therefore, the policy implications of studies will only be relevant to countries that utilise the healthcare model under study.

Given these caveats, there are some interesting findings of relevance to clinicians and policymakers. Looking at healthcare systems, better access to primary care is associated with lower rates of ED use and EHA in the USA, but this effect is not demonstrated in the UK and other European countries. The geographical location of services is important, with proximity to a general practice reducing unscheduled secondary care use and proximity to ED increasing usage. Convenience for patients therefore appears to be important, a finding that supports recent policy guidance in the UK. ${ }^{64}$ For practices, the impact of continuity of care with a primary care provider on both ED use and EHA is evident. This is a timely finding in view of the recent proposal in the UK to provide people over 75 years of age with a dedicated GP who is accountable for their care and who will be responsible for ensuring that their patients have good quality out of hours care. ${ }^{65}$

\section{Unanswered $\mathbf{Q}$ and future research}

The majority of research found was observational and this limits conclusions about how to change systems. While associations exist, such as the impact of increased continuity of care, this would not necessarily translate to reduced utilisation of USC if implemented. The current evidence base does not provide clear support for any particular policy change. It is clear that the decision to attend unscheduled care and the need to be admitted to hospital as an emergency are both the product of a complex interaction between individuals, their context, the organisation of healthcare, the behaviours of healthcare practitioners and the wider context of society. Further research needs to try to unpack in more nuanced detail the operation of these factors and the complex interactions between them.

Contributors AH is the main systematic reviewer involved in all stages of review and write-up. DL contributed to the methodological approach and also added significant input to the results and discussion. LW assisted with double data extraction and commented and advised on the write-up of the review. RM contributed to the methodological approach, advised on the statistical approach of individual papers and commented on the results and discussion. $\mathrm{HE}$ advised on the relevance of results to primary care practice and commissioning of care and also commented on the results and discussion. $\mathrm{KC}$ contributed to the significance of results to primary care and provided significant input to the results and discussion. CS contributed to the methodological approach and also added relevance to primary care as well as significant input to the written paper. SP is a PI on the original grant application and overall project; she was involved in all stages of the review including screening, data checking and write-up.

Funding This work was supported by the National School of Primary Care Research (NSPCR) grant funded round four, PI Sarah Purdy project number 115. This paper presents independent research funded by the National Institute for Health Research (NIHR). The views expressed are those of the authors and not necessarily those of the NHS, the NIHR or the Department of Health.

Competing interests DL is supported by the NIHR Oxford Biomedical Research Centre.

Provenance and peer review Not commissioned; externally peer reviewed.

Data sharing statement No additional data are available.

Open Access This is an Open Access article distributed in accordance with the Creative Commons Attribution Non Commercial (CC BY-NC 3.0) license, which permits others to distribute, remix, adapt, build upon this work noncommercially, and license their derivative works on different terms, provided the original work is properly cited and the use is non-commercial. See: http:// creativecommons.org/licenses/by-nc/3.0/

\section{REFERENCES}

1. NHS Healthcare for London. Commissioning a new delivery model for unscheduled care in London. Healthcare for London, 2011. http:// www.londonprogrammes.nhs.uk/wp-content/uploads/2011/03/ Unscheduled-care-commissioning-model.pdf (accessed 1 Dec 2013). 
2. NHS Improvement. Making connections with the challenges of unscheduled care. NHS Improvement, 2012. http://www.improvement nhs.uk/documents/Unscheduled_Care.pdf (accessed 1 Dec 2013).

3. Hallaran F, Roberston-steel I. A guide to good practice: unscheduled and emergency care services services. NLIAH Wales. http://www. wales.nhs.uk/sitesplus/documents/829/AGuide to Good Practice Unscheduled_and_Emergency_Care_Services.PDF (accessed 1 Dec 2013).

4. Tian Y. 2012 Data briefing: emergency hospital admissions for ambulatory care-sensitive conditions. Kings fund report. 3 April 2012. http://www.kingsfund.org.uk/publications/data-briefingemergency-hospital-admissions-ambulatory-care-sensitive-conditions (accessed 1 Dec 2013).

5. Lowthian JA, Curtis AJ, Cameron P, et al. Systematic review of trends in emergency department attendances: an Australian perspective. Emerg Med J 2011;28:373-7.

6. HES data. http://www.hscic.gov.uk/hes (accessed 1 Dec 2013)

7. Baker MJ, Bankart A, Rashid J, et al. Characteristics of general practices associated with emergency-department attendance rates: a cross-sectional study. BMJ Qual Saf 2011;20:953-8.

8. Saxena S, George J, Barber J, et al. Association of population and practice factors with potentially avoidable admission rates for chronic diseases in London: cross sectional analysis. $J R$ Soc Med 2006;99:81-9.

9. Aubin $M$, Giguère $A$, Martin $M$, et al. Interventions to improve continuity of care in the follow-up of patients with cancer. Cochrane Database Syst Rev 2012;CD007672.

10. Cabana MD, Jees $\mathrm{SH}$. Does continuity of care improve patient outcomes? J Fam Pract 2004;53:974-80.

11. Carret ML, Fassa AC, Domingues MR. Inappropriate use of emergency services: a systematic review of prevalence and associated factors. Cad Saude Publica 2009;25:7-28.

12. Flores-Mateo G, Violan-Fors C, Carrillo-Santisteve P, et al. Effectiveness of organizational interventions to reduce emergency department utilization: a systematic review. PLoS One 2012;7:e35903.

13. Hsiao CJ, Boult C. Effects of quality on outcomes in primary care: a review of the literature. Am J Med Qual 2008;23:302-10.

14. OECD website. http://www.oecd.org (accessed 1 Dec 2013).

15. CASP appraisal tool. http://www.casp-uk.net/ (accessed 1 Dec 2013)

16. Christakis DA, Mell L, Koepsell TD, et al. Association of lower continuity of care with greater risk of emergency department use and hospitalization in children. Pediatrics 2001;107:524-9.

17. Bankart MJ, Baker R, Rashid A, et al. Characteristics of general practices associated with emergency admission rates to hospital: a cross-sectional study. Emerg Med J 2011;28:558-63.

18. Cheung PT, Wiler JL, Lowe RA, et al. National study of barriers to timely primary care and emergency department utilization among Medicaid beneficiaries. Ann Emerg Med 2012;60:4-10.

19. Cheung PT, Wiler JL, Ginde AA. Changes in barriers to primary care and emergency department utilization. Arch Intern Med 2011;171:1397-9.

20. Dusheiko M, Doran T, Gravelle H, et al. Does higher quality of diabetes management in family practice reduce unplanned hospital admissions? Health Serv Res 2011;46(1 Pt 1):27-46.

21. Purdy S, Griffin T, Salisbury C, et al. Emergency respiratory admissions: influence of practice, population and hospital factors. $J$ Health Serv Res Policy 2011;16:133-40.

22. Purdy S, Griffin T, Salisbury C, et al. Emergency admissions for coronary heart disease: a cross-sectional study of general practice, population and hospital factors in England. Public health 2011;125:46-54.

23. Menec VH, Sirski M, Attawar D, et al. Does continuity of care with a family physician reduce hospitalizations among older adults? $J$ Health Serv Res Policy 2006;11:196.

24. Pereira AG, Kleinman KP, Pearson SD. Leaving the practice: effects of primary care physician departure on patient care. Arch Intern Med 2003;163:2733-6.

25. Bottle A, Millett C, Xie Y, et al. Quality of primary care and hospital admissions for diabetes mellitus in England. $J$ Ambul Care Manage 2008:31:226-38.

26. Stern Z, Calderon-Margalit R, Mazar M, et al. Emergency room visit: a red-flag indicator for poor diabetes care. Diabet Med 2009;26:1105-11.

27. Brousseau DC, Gorelick MH, Hoffmann RG, et al. Primary care quality and subsequent emergency department utilization for children in Wisconsin Medicaid. Acad Pediatr 2009:9:33-9.

28. Brousseau DC, Hoffmann RG, Nattinger AB, et al. Quality of primary care and subsequent pediatric emergency department utilization. Pediatrics 2007;119:1131-8.
29. Burge F, Lawson B, Johnston G. Family physician continuity of care and emergency department use in end-of-life cancer care. Med Care 2003;41:992-1001.

30. Thomas DP, Anderson IP, Kelaher MA. Accessibility and quality of care received in emergency departments by Aboriginal and Torres Strait Islander people. Aust Health Rev 2008;32:648-54.

31. Thompson C, Hayhurst C, Boyle A. How have changes to out-ofhours primary care services since 2004 affected emergency department attendances at a UK District General Hospital? A longitudinal study. Emerg Med J 2010;27:22-5.

32. Solberg LI, Maciosek MV, Sperl-Hillen JM, et al. Does improved access to care affect utilization and costs for patients with chronic conditions? Am J Manage Care 2004;10:717-22.

33. Begley CE, Vojvodic RW, Seo M, et al. Emergency room use and access to primary care: evidence from Houston, Texas. J Health Care Poor Underserved 2006;17:610-24.

34. Carlsen F, Grytten J, Kjelvik J, et al. Better primary physician services lead to fewer hospital admissions. Eur J Health Econ 2007;8:17-24

35. Ionescu-Ittu R, McCusker J, Ciampi A, et al. Continuity of primary care and emergency department utilization among elderly people. CMAJ 2007;177:1362-8.

36. Duffy $R$, Neville R, Staines $H$. Variance in practice emergency medical admission rates: can it be explained? $\mathrm{Br} J$ Gen Pract 2002;52:14-7.

37. Rizza P, Bianco A, Pavia M, et al. Preventable hospitalization and access to primary health care in an area of Southern Italy. BMC Health Serv Res 2007:7:134.

38. Basu J, Friedman B, Burstin H. Primary care, HMO enrolment, and hospitalization for ambulatory care sensitive conditions-a new approach. Med Care 2002;40:1260-9.

39. Cowling TE, Cecil EV, Soljak MA, et al. Access to primary care and visits to emergency departments in England: a cross-sectional, population-based study. PLoS One 2013;8:e66699.

40. Harris MJ, Patel B, Bowen S. Primary care access and its relationship with emergency department utilisation: an observational, cross-sectional, ecological study. BJGP 2011;61:e787-93.

41. Majeed A, Bardsley M, Morgan D, et al. Cross sectional study of primary care groups in London: association of measures of socioeconomic and health status with hospital admission rates. BMJ 2000;321:1057-60.

42. Downing A, Rudge G, Cheng Y, et al. Do the UK government's new Quality and Outcomes Framework (QOF) scores adequately measure primary care performance? A cross-sectional survey of routine healthcare data. BMC Health Serv Res 2007;7:166.

43. Lowe RA, Localio AR, Schwarz DF, et al. Association between primary care practice characteristics and emergency department use in a Medicaid managed care organization. Med Care 2005:43:792-800.

44. Sturm JJ, Hirsh DA, Lee EK, et al. Practice characteristics that influence non-urgent pediatric emergency department utilization. Acad Pediatr 2010;10:70-4

45. Magan $\mathrm{P}$, Alberquilla A, Otero A, et al. Hospitalizations for ambulatory care sensitive conditions and quality of primary care thei relation with socioeconomic and health care variables in the Madrid Regional Health Service (Spain). Med Care 2011;49:17-23.

46. Ricketts TC, Randolph R, Hilda Ann Howard HA, et al. Hospitalization rates as indicators of access to primary care. Health Place 2001;7:27-38.

47. Lowe RA, Fu R, Ong ET, et al. Community characteristics affecting emergency department use by Medicaid enrollees. Med Care 2009;47:15-22.

48. van Uden CJ, Crebolder HF. Does setting up out of hours primary care cooperatives outside a hospital reduce demand for emergency care? Emerg Med J 2004;21:722-3.

49. Vedsted P, Christensen MB. The effect of an out-of-hours reform on attendance at casualty wards. The Danish example. Scand J Prim Health Care 2001:19:95-8.

50. de la Fuente $\mathrm{D}$, Pino $\mathrm{J}$, Blanco $\mathrm{V}$, et al. Does better access to primary care reduce utilization of hospital accident and emergency departments? A time-series analysis. Eur J Public Health 2007;17:186-92.

51. McCusker J, Roberge D, Lévesque JF, et al. Emergency department visits and primary care among adults with chronic conditions. Med Care 2010;48:972-80.

52. McCusker J, Tousignant $P$, Borgès Da Silva $R$, et al. Factors predicting patient use of the emergency department: a retrospective cohort study. CMAJ 2012;184:E307-16.

53. Ludwick A, Fu R, Warden C, et al. Distances to emergency department and to primary care provider's office affect 
emergency department use in children. Acad Emerg Med 2009;16:411-17.

54. Gill JM, Mainous AG III, Nsereko M. The effect of continuity of care on emergency department use. Arch Fam Med 2000;9:333-8.

55. Hossain M, Laditka JN. Using hospitalization for ambulatory care sensitive conditions to measure access to primary health care: an application of spatial structural equation modelling. Int $J$ Health Geograph 2009;8:51.

56. Nolan A. An extension in eligibility for free primary care and avoidable hospitalisations: a natural experiment. Soc Sci Med 2011;73:978-e985.

57. Gulliford MC. Availability of primary care doctors and population health in England: is there an association? J Public Health Med 2002;24:252-4.

58. Hull S, Harvey C, Sturdy P, et al. Do practice-based preventive child health services affect the use of hospitals? A cross-sectional study of hospital use by children in east London. BJGP 2000;50:31-6.

59. Kronman AC, Ash AS, Freund KM, et al. Can primary care visits reduce hospital utilization among medicare beneficiaries at the end of life? J Gen Intern Med 2008;23:1330-5.
60. Pines JM, Mutter RL, Pilgrim R, et al. Variation in emergency department admission rates across the United States. Med Care Res Rev 2013;70:218-31.

61. Reid RJ, Johnson EA, Hsu C, et al. Spreading a medical home redesign: effects on emergency department use and hospital admissions. Ann Fam Med 2013;11(Suppl 1):S19-26.

62. Gunther S, Taub N, Rogers S, et al. What aspects of primary care predict emergency admission rates? A cross sectional study. BMC Health Serv Res 2013;11-3.

63. Brettell R, Soljak M, Cecil E, et al. Reducing heart failure admission rates in England 2004-2011 are not related to changes in primary care quality: national observational study. Eur J Heart Fail 2013;15:1335-42.

64. NHS England. High quality care for all, now and for future generations: transforming urgent and emergency care services in England-Urgent and Emergency Care Review End of Phase 1 Report November 2013. http://www.nhs.uk/NHSEngland/keogh-review/Documents/UECR. Ph1Report.FV.pdf) (accessed 1 Dec 2013)

65. Department of Health. Personalised GP care will bring back oldfashioned family doctors. November 2013. http://www.gov.uk/ government/news/personalised-gp-care-will-bring-back-oldfashionedfamily-doctors (accessed 1 Dec 2013) 